\title{
THE MOTION OF A SYSTEM OF COAXIAL BODIES OF VARIABLE MASS $\dagger$
}

\author{
V. S. ASLANOV and A. V. DOROSHIN \\ Samara \\ e-mail: aslanov@ssau.ru \\ (Received 26 December 2002)
}

\begin{abstract}
The motion of a mechanical system of coaxial axisymmetrical bodies of variable mass in a translating system of coordinates is considered. A theorem on the change in the angular momentum of a system of coaxial bodies of variable mass with respect to translating axes is given. The dynamic equations of motion are constructed using the example of two coaxial bodies. Assuming that the relative displacements of the centre of mass, due to a change in the mass of the system, are small, approximate solutions are found for the spatial orientation angles and the condition for reducing the amplitude of nutational oscillations. The results obtained can be used to describe the motion of spacecraft, constructed in coaxial form, when performing active manoeuvres with a change in mass. (c) 2005 Elsevier Ltd. All rights reserved.
\end{abstract}

The motion of a spacecraft with double rotation and fixed mass when there is a small asymmetry was considered in a previous paper [1].

\section{FORMULATION OF THE PROBLEM AND A THEOREM ON THE CHANGE IN THE ANGULAR MOMENTUM OF A SYSTEM OF COAXIAL BODIES}

We will consider the problems of deriving the equations of motion of a system of $k$ bodies of variable mass with respect to translating axes and of obtaining approximate analytical relations for a free system of two coaxial bodies. The dynamic symmetry is not disturbed during the change in mass.

We will introduce the following system of coordinates (Fig. 1): $P \xi \eta \zeta$ is a system of coordinates, fixed in absolute space, $O X Y Z$ is a moving system of coordinates with origin at the point $O$, the axes of which remain collinear with the axes of the fixed system during the whole time of motion, and $O x_{i} y_{i} z_{i}$ are systems of coordinates with a common origin, rigidly connected to the $i$-th body $(i=1,2, \ldots, k)$, rotating with respect to the system $O X Y Z$. We will choose as the origin of coordinates of $O X Y Z$ a point lying on the common axis of rotation of the bodies and coinciding with the initial position of the centre of mass, Points forming part of the system are distinguished by the fact that they belong to one or other body, and hence when writing expressions we will indicate that points belong to the $i$ th body by the subscript $v_{i}$.

To construct the equations of motion we will use the "short-range" hypothesis, according to which particles which obtain a relative velocity when separated from the body no longer belong to the body and in no way act on it, in which case the theorem on the change in the angular momentum of a system of variable mass [2], written with respect to the fixed system of coordinates $P \xi \eta \zeta$, takes the form

$$
\frac{d \mathbf{K}_{P}}{d t}=\mathbf{M}_{P}^{e}+\mathbf{M}_{P}^{R}+\sum_{i=1}^{k} \mathbf{S}_{i}^{e}, \quad \mathbf{S}_{i}^{e}=\sum_{v_{i}} \mathbf{r}_{v_{i}} \times \frac{d m_{v_{i}}}{d t} \mathbf{v}_{v_{i}}
$$

$\dagger$ Prikl. Mat. Mekh. Vol. 68, No. 6, pp. 999-1009, 2004.

0021-8928/\$-see front matter. (C) 2005 Elsevier Ltd. All rights reserved.

doi: 10.1016/j.jappmathmech.2004.11.012 


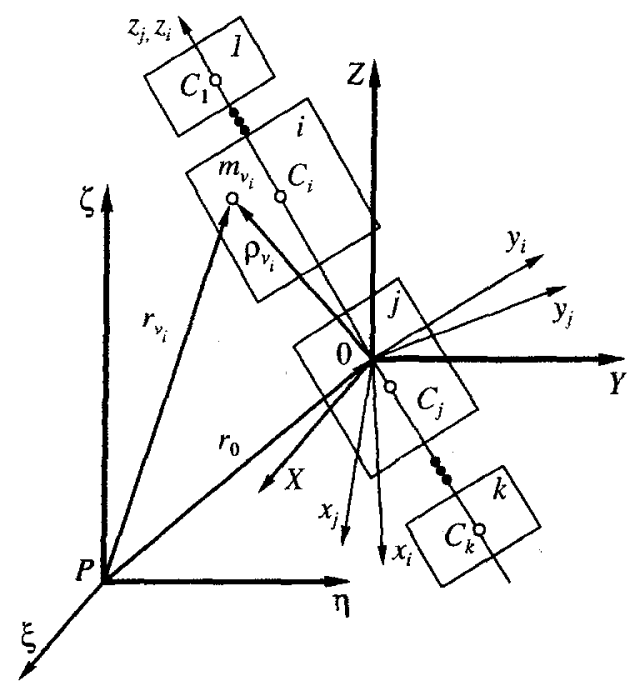

Fig. 1

where $\mathbf{M}_{P}^{e}$ is the principal moment of the external forces, $\mathbf{M}_{P}^{R}$ is the principal moment of the reactive forces, and $\mathbf{S}_{i}^{e}$ is the sum of the angular momenta of the particles of body $i$, rejected in unit time in their translational motion with respect to the fixed system of coordinates.

The angular momentum of a system of $k$ bodies in the system of coordinates OXYZ (Fig. 1) is given by the formula

$$
\mathbf{K}_{P}=\sum_{i=1}^{k} \sum_{v_{i}} \mathbf{r}_{v_{i}} \times m_{v_{i}} \mathbf{v}_{v_{i}}=\sum_{i=1}^{k} \sum_{v_{i}}\left[\left(\mathbf{r}_{0}+\boldsymbol{\rho}_{v_{i}}\right) \times m_{v_{i}}\left(\mathbf{v}_{0}+\boldsymbol{\omega}_{i} \times \boldsymbol{\rho}_{v_{i}}\right)\right]
$$

where $\omega_{i}$ is the absolute angular velocity of body $i$ and the system of coordinates $O x_{i} y_{i} z_{i}$ connected with it.

To write the theorem of the change is the angular momentum with respect to the moving system of coordinates $O X Y Z$, we will use the idea of a centre of mass for each body and write the auxiliary relations

$$
\begin{aligned}
& \frac{d \boldsymbol{\rho}_{C_{i}}}{d t}=\boldsymbol{\omega}_{i} \times \boldsymbol{\rho}_{C_{i}}+\mathbf{q}_{C_{i}} \\
& \sum_{v_{i}} \frac{d m_{v_{i}}}{d t}\left(\boldsymbol{\omega}_{i} \times \boldsymbol{\rho}_{v_{i}}\right)=\boldsymbol{\omega}_{i} \times\left[\frac{d m_{i}}{d t} \boldsymbol{\rho}_{C_{i}}+m_{i} \mathbf{q}_{C_{i}}\right]
\end{aligned}
$$

where $\rho_{C_{i}}$ is the radius vector of the centre of mass $C_{i}$ of body $i$ in the system $O X Y Z$ and $\mathbf{q}_{C_{i}}$ is the relative velocity of the centre of mass $C_{i}$, due to a change in its position with respect to the bodies, due to the variability of their masses. If we use these relations and group the points of the system in accordance with their membership of the bodies $i$, we can write the theorem on the change in angular momentum with respect to the system of axes $O X Y Z$ in the form

$$
\sum_{i=1}^{k} \frac{d \mathbf{K}_{i, o}}{d t}=\mathbf{M}_{O}^{e}+\mathbf{M}_{O}^{R}+\sum_{i=1}^{k} \sum_{v_{i}} \boldsymbol{\rho}_{v_{i}} \times \frac{d m_{v_{i}}}{d t}\left(\boldsymbol{\omega}_{i} \times \boldsymbol{\rho}_{v_{i}}\right)-\boldsymbol{\rho}_{C} \times m \mathbf{w}_{O}
$$

where $\mathbf{M}_{O}^{e}$ and $\mathbf{M}_{O}^{R}$ are the principal moments of the external and reactive forces with respect to the point $O$. Expression (1.3) corresponds to the assertion of the well-known theorem [2], taking into account the grouping of the terms according to the membership of the points of the body $i(i=1, \ldots, k)$.

Using the idea of a local derivative for the angular momentum vector of each body in the system of coordinates $O x_{i} y_{i} z_{i}$ connected with the body, rotating with respect to $O X Y Z$ with angular velocity $\omega_{i}$, Eq. (1.3) can be rewritten as follows: 


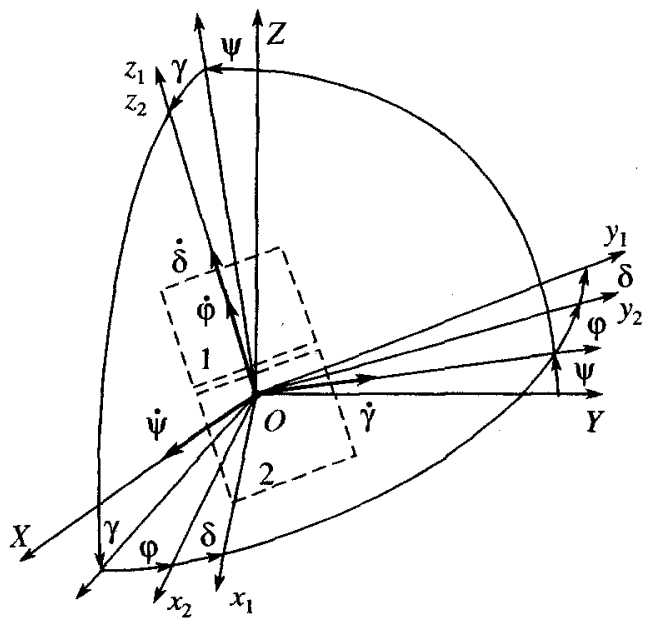

Fig. 2

$$
\begin{aligned}
& \sum_{i=1}^{k}\left[\left(\frac{\tilde{d} \mathbf{K}_{i, O}}{d t}\right)_{O x_{i} y_{i} z_{i}}+\boldsymbol{\omega}_{i} \times \mathbf{K}_{i, O}\right]= \\
& =\mathbf{M}_{O}^{e}+\mathbf{M}_{O}^{R}+\sum_{i=1}^{k} \sum_{\mathbf{v}_{t}} \boldsymbol{\rho}_{v_{t}} \times \frac{d m_{\mathbf{v}_{i}}}{d t}\left(\boldsymbol{\omega}_{i} \times \boldsymbol{\rho}_{v_{i}}\right)-\boldsymbol{\rho}_{C} \times m \mathbf{w}_{O}
\end{aligned}
$$

We have indicated in the subscript outside the brackets of the local derivatives the systems of coordinates in which they are taken.

Equation (1.4) expresses in vector form the theorem of the change in the angular momentum of bodies of variable mass with respect to the translating axes.

\section{A SYSTEM OF TWO COAXIAL BODIES}

We will consider the free motion of a system of two dynamically symmetrical bodies, where only body 1 is of variable mass. The rejecting of point masses, when there is a change in mass, occurs symmetrically, so that the vector of the reactive forces is directed strictly along the axis of rotation. Body 2 does not change its inertia-mass characteristics, calculated in the system of coordinates $O x_{2} y_{2} z_{2}$ connected to the body, and, consequently, produces no reactive forces. The centre of mass of the system, due to the change in the mass of body 1 , is shifted with a certain velocity $\mathbf{q}_{C}$ strictly along the longitudinal axis. In Fig. 2 we show the case when, at the initial instant of time, the mass of the second body is greater than the mass of the first one.

We will write the angular velocities and the angular momenta of the bodies in projections onto the axes of their connected systems of coordinates

$$
\begin{gathered}
\boldsymbol{\omega}_{i}=p_{i} \mathbf{i}_{i}+q_{i} \mathbf{j}_{i}+r_{i} \mathbf{k}_{i} \\
\mathbf{K}_{1, o}=A_{1}(t)\left(p_{1} \mathbf{i}_{1}+q_{1} \mathbf{j}_{1}\right)+C_{1}(t) r_{1} \mathbf{k}_{1}, \quad \mathbf{K}_{2, o}=A_{2}\left(p_{2} \mathbf{i}_{2}+q_{2} \mathbf{j}_{2}\right)+C_{2} r_{2} \mathbf{k}_{2}
\end{gathered}
$$

where $A_{i}$ and $C_{i}$ are the equatorial and longitudinal moments of inertia of body $i$, calculated in the corresponding system of coordinates connected to the body, and $\left\{\mathbf{i}_{i}, \mathbf{j}_{i}, \mathbf{k}_{i}\right\}$ are the unit vectors of the system $O x_{i} y_{i} z_{i}(i=1,2)$.

The bodies of the system can only rotate with respect to one another in the direction of the common longitudinal axis, which coincides with $O z_{2}$ (and which $O z_{1}$ ). Here we will denote the angle and velocity of twisting of body 1 with respect to body 2 in the direction of the longitudinal axis $O z_{2}$ by $\delta$ and $\sigma=\dot{\delta}$ respectively. The angles of spatial orientation of the coaxial bodies with respect to the translating system of coordinates $O X Y Z$ are indicated in Fig. 2. The relation between the angular velocities and the angular accelerations of two bodies in vector form is given by the formulae

$$
\boldsymbol{\omega}_{1}=\omega_{2}+\boldsymbol{\sigma}, \quad \varepsilon_{1}=\varepsilon_{2}+\dot{\sigma}
$$



From the theorem on the motion of the centre of mass of a system of variable mass [2] and expression (2.3), the following relation must hold

$$
m \mathbf{w}_{O}=\boldsymbol{\Phi}_{1}^{R}-\varepsilon_{2} \times m \boldsymbol{\rho}_{C}-m \omega_{2} \times \omega_{2} \times \boldsymbol{p}_{C}
$$

Bearing in mind that $\boldsymbol{\rho}_{C}=\left(0,0, \rho_{C}\right), \boldsymbol{\rho}_{C} \times \boldsymbol{\omega}_{2} \times \boldsymbol{\omega}_{2} \times \boldsymbol{\rho}_{C}=0$ and also (2.9), using Eq. (2.10) we will represent the vector $\left(-\boldsymbol{\rho}_{C} \times m \mathrm{w}_{O}\right)$ in terms of projections onto the axes of system $O x_{2} y_{2} z_{2}$

$$
-\left[m \boldsymbol{\rho}_{C} \times \mathbf{w}_{O}\right]=m \rho_{C}^{2}\left[\dot{p} \dot{\mathbf{i}}_{2}+\dot{q} \mathbf{j}_{2}+0 \mathbf{k}_{2}\right]
$$

Taking relation (2.11) into account, we will write Eqs (2.8) in the form

$$
\begin{aligned}
& \left(A_{3}(t)-m \rho_{C}^{2}(t)\right) \dot{p}_{2}+\left(C_{3}(t)-A_{3}(t)\right) q_{2} r_{2}+C_{1}(t) q_{2} \sigma=0 \\
& \left(A_{3}(t)-m \rho_{C}^{2}(t)\right) \dot{q}_{2}-\left(C_{3}(t)-A_{3}(t)\right) p_{2} r_{2}-C_{1}(t) p_{2} \sigma=0 \\
& C_{3}(t) \dot{r}_{2}+C_{1}(t) \dot{\sigma}=0
\end{aligned}
$$

where $\rho_{C}(t)$ is a known function of time.

We will add to the three dynamic equations (2.12) an equation which describes the relative motion of the bodies. We will use the theorem on the change in the angular momentum in terms of the projection onto the axis of rotation, written for the first body

$$
\frac{d K_{z_{1}}}{d t}=M_{\delta}+\left(\sum_{v_{1}} \boldsymbol{\rho}_{v_{1}} \times \frac{d m_{v_{1}}}{d t} \dot{\boldsymbol{\rho}}_{v_{1}}\right)_{z_{1}}
$$

where $K_{z_{1}}=C_{1}(t) r_{1}$ is the projection of the angular momentum of the first body onto the axis of rotation and $M_{\delta}$ is the moment of the internal interaction of the bodies. Since the centrifugal moments of inertia of the body are zero, this equation takes the form

$$
C_{1}(t)\left(\dot{r}_{2}+\dot{\sigma}\right)=M_{\delta}
$$

We will supplement the dynamic equations (2.12) and (2.13) by the following kinematic relations (Fig. 2)

$$
\begin{aligned}
& \dot{\gamma}=p_{2} \sin \varphi+q_{2} \cos \varphi, \quad \dot{\psi}=\frac{1}{\cos \gamma}\left(p_{2} \cos \varphi-q_{2} \sin \varphi\right) \\
& \dot{\varphi}=r_{2}-\frac{\sin \gamma}{\cos \gamma}\left(p_{2} \cos \varphi-q_{2} \sin \varphi\right), \quad \dot{\delta}=\sigma
\end{aligned}
$$

If, when the mass changes, the quantity $m \rho_{C}^{2}(t)$ remains small compared with the overall transverse moment of inertia of the system $A_{3}(t)$, the system of dynamic equations takes the form

$$
\begin{aligned}
& A_{3}(t) \dot{p}_{2}+\left(C_{3}(t)-A_{3}(t)\right) q_{2} r_{2}+C_{1}(t) q_{2} \sigma=0 \\
& A_{3}(t) \dot{q}_{2}-\left(C_{3}(t)-A_{3}(t)\right) p_{2} r_{2}-C_{1}(t) p_{2} \sigma=0 \\
& C_{3}(t) \dot{r}_{2}+C_{1}(t) \dot{\sigma}=0, \quad C_{1}(t)\left(\dot{r}_{2}+\dot{\sigma}\right)=M_{\delta}
\end{aligned}
$$

It follows from system (2.15) that the equations of motion of coaxial bodies of variable mass, in the case of small relative displacements of centre of mass, differ from the equations of motion of coaxial bodies of constant mass [1] in the fact that the moments of inertia vary with time. When the velocity of relative twisting is identically equal to zero $(\sigma=\dot{\delta} \equiv 0)$, Eqs $(2.15)$ are identical with the well-known equations of the free motion of a dynamically symmetrical body of variable composition $[2,3]$.

\section{SOLUTIONS FOR THE ANGLES OF ORIENTATION OF A COAXIAL SYSTEM OF TWO BODIES}

Suppose there is no moment of internal interaction between the coaxial bodies $\left(M_{\delta} \equiv 0\right)$, the final displacement of the centre of mass is small, and the equatorial and longitudinal moments of inertia of body 1 vary linearly 


$$
A_{1}(t)=A_{1}-a t, \quad C_{1}(t)=C_{1}-c t ; \quad a=\left(A_{1}-A_{1 f}\right) / T, \quad c=\left(C_{1}-C_{1 f}\right) / T
$$

where $A_{1}, C_{1}, A_{1 f}, C_{1 f}$ are quantities corresponding to the beginning and end of mass change process, and $T$ is its duration.

We will introduce new dimensionless variables $G$ and $F$ by the following replacement

$$
p_{2}(t)=\omega G(t) \sin F(t), \quad q_{2}(t)=\omega G(t) \cos F(t)
$$

where $\omega=\left(r_{0}\left(A_{1}+A_{2}-C_{1}-C_{2}\right)-C_{1} \sigma_{0}\right) /\left(A_{1}+A_{2}\right)$ is the characteristic velocity. The variable $G$ in formulae (3.2) is the dimensionless transverse angular velocity of the system of bodies

$$
G(t)=\left(p_{2} \sin F+q_{2} \cos F\right) / \omega
$$

while the variable phase $F$ defines the angle between the transverse angular velocity vector and the $O y$ axis.

System (2.15) can be written in terms of the new variables as follows:

$$
\dot{G}=0, \quad(A-a t) \dot{F}=A \omega-n t ; \quad r_{2}=r_{0}, \quad \sigma=\sigma_{0}\left(r_{1}=r_{0}+\sigma_{0}\right)
$$

where

$$
A=A_{1}+A_{2}, \quad n=a r_{0}-c\left(r_{0}+\sigma_{0}\right)
$$

System (3.3) has the exact solution

$$
G=L_{0}, \quad F(t)=s_{0}+\frac{n}{a} t-\frac{A}{a^{2}}(a \omega-n) \ln \left(1-\frac{a}{A} t\right)
$$

where $L_{0}$ and $s_{0}$ are the initial values of the amplitude and phase.

Suppose the ratio of the change in the equatorial moment of inertia of body 1 to the initial equatorial moment of inertia of the system is a small quantity

$$
\mu=\left(A_{1}-A_{1 f}\right) /\left(A_{1}+A_{2}\right)=a T / A \ll 1
$$

By representing the natural logarithm in solution (3.4) in the form of a power series, which converges over the whole time interval, and dropping quantities of the order of $\mu^{2}$ and higher, we can write the solution for the phase

$$
F(t)=s_{0}+\omega t+\kappa t^{2}, \quad \kappa=(a \omega-n) /(2 A)
$$

Using expression (3.5) for the equatorial angular velocities we can write the following solutions

$$
p_{2}(t)=\omega L_{0} \sin \left((\omega+\kappa t) t+s_{0}\right), \quad q_{2}(t)=\omega L_{0} \cos \left((\omega+\kappa t) t+s_{0}\right)
$$

We will consider the case when body 1 rotates rapidly, body 2 is fixed with respect to the longitudinal axis $\left(r_{0}=0\right)$ and the modulus of the transverse angular velocity of the system is small compared with the characteristic angular velocity

$$
\varepsilon=\sqrt{p_{2}^{2}+q_{2}^{2}} /|\omega|=|G|=\left|L_{0}\right| \ll 1
$$

The angles of orientation $\gamma$ and $\psi$ will be assumed to be small $(\gamma=O(\varepsilon), \psi=O(\varepsilon))$. Then the nutation angle $\theta$ (the angle between the $O Z$ and $O z_{i}$ axes) are given by the following approximate formulae

$$
\theta^{2} \cong \gamma^{2}+\psi^{2}
$$

Taking relations (3.5), (3.7) and (3.8) into account, we can write kinematic equations (2.14) in the form

$$
\dot{\gamma}=\omega L_{0} \sin (F(t)+\varphi), \quad \dot{\psi}=\omega L_{0} \cos (F(t)+\varphi), \quad \dot{\varphi}=-\gamma \omega L_{0} \cos (F(t)+\varphi)
$$


In view of the above assumptions, the quantity $\dot{\varphi}$ is of higher order of smallness than $\dot{\gamma}$ and $\dot{\psi}$, and hence we can assume that, over a small time interval $\varphi=$ const $=0$. Then, we can write for the angular velocities $\dot{\gamma}$ and $\dot{\psi}$

$$
\dot{\gamma}=\omega L_{0} \sin F(t), \quad \dot{\psi}=\omega L_{0} \cos F(t)
$$

We will consider two possible cases of the motion, which occur for the following relations between the quantities

$$
\text { 1) } \operatorname{sign} \omega=\operatorname{sign} \kappa, 2) \operatorname{sign} \omega=-\operatorname{sign} \kappa
$$

Assuming, to fix our ideas, that the value of the frequency $\omega$ is positive, for both cases of (3.10), using Fresnel integrals, we can write the following analytic relations for the angles of orientation of the system (the upper plus and minus signs are taken for case 1, and the lower signs are taken for case 2)

$$
\begin{aligned}
& \gamma(t)= \pm c_{\mp}[S(\lambda(t))-S(\lambda(0))]+s_{\mp}[C(\lambda(t))-C(\lambda(0))]+\gamma_{0} \\
& \psi(t)=c_{\mp}[C(\lambda(t))-C(\lambda(0))] \mp s_{\mp}[S(\lambda(t))-S(\lambda(0))]+\psi_{0}
\end{aligned}
$$

where

$$
\begin{aligned}
& c_{ \pm}=R \cos \left(s_{0} \pm \frac{\omega^{2}}{4 \kappa}\right), \quad s_{ \pm}=R \sin \left[s_{0} \pm \frac{\omega^{2}}{4 \kappa}\right], \quad \lambda(t)=\sqrt{\frac{2|\kappa|}{\pi}}\left(t+\frac{\omega}{2 \kappa}\right), \quad R=L_{0} \sqrt{\frac{\pi}{2|\kappa|}} \\
& C(x)=\int_{0}^{x} \cos \left(\frac{\pi}{2} x^{2}\right) d x, \quad S(x)=\int_{0}^{x} \sin \left(\frac{\pi}{2} x^{2}\right) d x
\end{aligned}
$$

$(C(x)$ and $S(x)$ are Fresnel integrals).

\section{ANALYSIS OF THE AMPLITUDE OF NUTATIONAL OSCILLATIONS FOR SMALL CHANGES IN THE MOMENTS OF INERTIA}

We will consider the motion of a spacecraft, consisting of two coaxial bodies, one of which is a braking motor - body 1 . Body 2 does not rotate $\left(r_{0}=0\right)$ and its mass is fixed, while body 1 is of variable mass which twists with respect to the second body $\left(\sigma_{0} \neq 0\right)$. This arrangement can be used to slow down a spacecraft when it enters the atmosphere [1]. It is obvious that one must try to ensure that, during the braking process, the longitudinal axis of the system retains its position in space. We will obtain an estimate of the nutation angle at the braking stage. When $r_{0}=0$ formula (3.5), takes the form

$$
\kappa=\omega \frac{a C_{1}-c A}{2 C_{1} A}
$$

We will consider the case when the final changes in the values of the equatorial and longitudinal moments of body 1 , referred to the overall equatorial moment of inertia of the system and to the initial longitudinal moment of inertia of body 1 , respectively, are small:

$$
\mu=\Delta_{A} / A \ll 1, \quad \Delta_{C} / C_{1} \ll 1
$$

where $\Delta_{A}=A_{1}-A_{1 f}, \Delta_{C}=C_{1}-C_{1 f}$ are positive finite changes in the values of the corresponding moments of inertia of body 1. Limitations (3.7), (3.8) and (4.2) correspond to the practical problem of slowing down a spacecraft using a motor (the duration $T$ of the braking process does not exceed $30 \mathrm{~s}$ ).

We will introduce the instantaneous frequency

$$
\Omega(t)=\omega+\kappa t=\omega(1+\tau(t)), \quad \tau(t)=\frac{a C_{1}-c A}{2 C_{1} A} t
$$

Equations (3.9) then take the form

$$
\dot{\gamma}=\omega L_{0} \sin \left(\Omega(t) t+s_{0}\right), \quad \dot{\psi}=\omega L_{0} \cos \left(\Omega(t) t+s_{0}\right)
$$


The greatest value in modulus of $\tau(t)$ will be the small quantity

$$
\underset{t}{\sup }|\tau(t)|=\left|\frac{a C_{1}-c A}{2 C_{1} A}\right| T=\frac{1}{2}\left|\frac{\Delta_{A}}{A}-\frac{\Delta_{C}}{C_{1}}\right| \ll 1
$$

We can obtain an approximate representation of the motion of the system [4] if we take $\tau(t)$ as the parameter, assuming it to be equal to its mean value

$$
\bar{\tau}=\frac{1}{4}\left(\frac{\Delta_{A}}{A}-\frac{\Delta_{C}}{C_{1}}\right)
$$

In this case the approximate solutions of Eqs (4.3) have the form

$$
\begin{aligned}
& \gamma(t) \approx-\frac{L_{0}}{1+\bar{\tau}}\left[\cos \left(\bar{\Omega} t+s_{0}\right)-\cos s_{0}\right]+\gamma_{0} \\
& \psi(t) \approx \frac{L_{0}}{1+\tilde{\tau}}\left[\sin \left(\bar{\Omega} t+s_{0}\right)-\sin s_{0}\right]+\Psi_{0} \\
& \bar{\Omega}=\omega(1+\bar{\tau})
\end{aligned}
$$

The following relation for the time dependence of the nutation angle follows from expressions (3.8) and (4.4)

$$
\begin{aligned}
& \theta^{2}(t)=\frac{2 L_{0}^{2}}{(1+\bar{\tau})^{2}}[1-\cos (\bar{\Omega} t)]+\frac{2 L_{0}}{1+\bar{\tau}}\left\{\psi_{0}\left(\sin \left(\bar{\Omega} t+s_{0}\right)-\sin s_{0}\right)-\right. \\
& \left.-\gamma_{0}\left(\cos \left(\bar{\Omega} t+s_{0}\right)-\cos s_{0}\right)\right\}+\theta_{0}^{2}, \quad \theta_{0}^{2}=\gamma_{0}^{2}+\psi_{0}^{2}
\end{aligned}
$$

Averaging over the fast phase $\chi=\bar{\Omega} t$ we obtain the following approximate formula

$$
\left\langle\theta^{2}\right\rangle=\frac{2 L_{0}^{2}}{(1+\bar{\tau})^{2}}+\frac{2 L_{0}}{1+\bar{\tau}} D+\theta_{0}^{2}
$$

where

$$
D=\sqrt{\gamma_{0}^{2}+\psi_{0}^{2}} \cos \left(\alpha+s_{0}\right), \quad \cos \alpha=\frac{\gamma_{0}}{\sqrt{\gamma_{0}^{2}+\psi_{0}^{2}}}, \quad \sin \alpha=\frac{\psi_{0}}{\sqrt{\gamma_{0}^{2}+\psi_{0}^{2}}}
$$

We will consider the special case when $D=0$, which occurs when $\gamma_{0}=\psi_{0}$ (when $\alpha=\pi / 4$ ) and when $p_{0}=q_{0}$ (when $s_{0}=\pi / 4$ ), which can always be achieved by an appropriate choice of the systems of coordinates. It then follows from (4.5) that, to reduce the mean value of the nutation angle, it is necessary to increase the sum $1+\bar{\tau}$, which is equivalent to the following conditions

$$
\bar{\tau}>0, \quad|\bar{\tau}| \rightarrow \sup
$$

The characteristic frequency $\omega$ in this case has the following value

$$
\omega=-C_{1} \sigma_{0} / A
$$

The first condition of (4.6) is equivalent to the inequality

$$
\Delta_{A} / A>\Delta_{C} / C_{1}
$$

The second condition of (4.6) for a fixed value of the velocity of relative twisting of the bodies $\sigma_{0}$ and, as a consequence, for a fixed value of the characteristic frequency $\omega(4.7)$, reduces to the condition for the modulus of $\bar{\tau}$ to increase and takes the form

$$
\Delta_{A} / A-\Delta_{C} / C_{1} \rightarrow \sup _{\left\{\Delta_{A}, \Delta_{C}\right\}}
$$




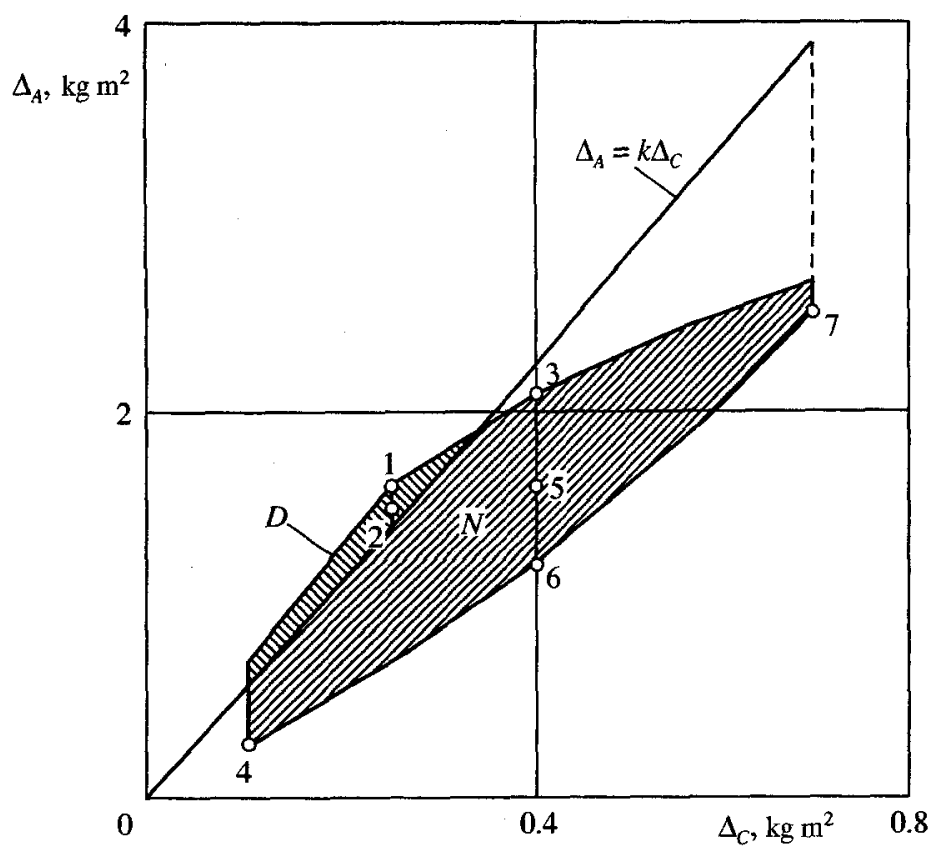

Fig. 3

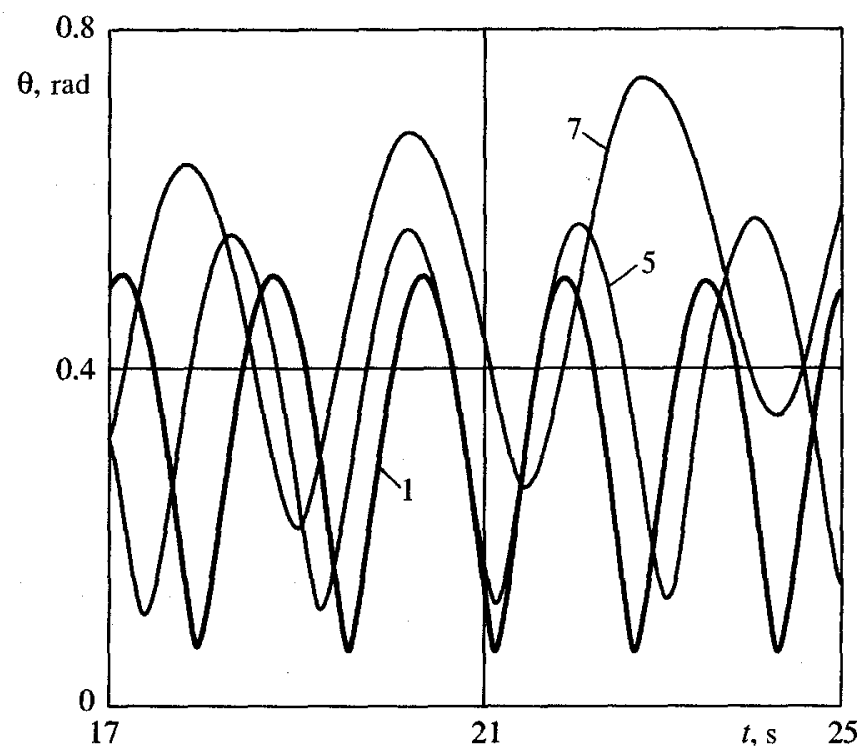

Fig. 4

In Fig. 3 in the spatial variables $\left\{\Delta_{A}, \Delta_{C}\right\}$ we have represented the straight line

$$
\Delta_{A}=k \Delta_{C}, \quad k=A / C_{1}
$$

which defines the boundary, above which (the region $D$ in Fig. 3) condition (4.8) is satisfied, and below which (the region $N$ in Fig. 3) it is not satisfied. When considering the practical example of the braking of a spacecraft these region $N$ and $D$ represent a subset of sets of possible design parameters. These design parameters are the final changes in the moments of inertia of the braking motor $\left\{\Delta_{A}, \Delta_{C}\right\}$, defining the form and internal arrangement of the fuel charges - the solid-fuel packets of the channel burner.

The points $\left\{\Delta_{A}, \Delta_{C}\right\}$, situated above the straight line (4.10) and furthest from it, satisfy condition (4.9). In Fig. 3 we show a set of points, corresponding to the regions of possible design parameters for a spacecraft, which are numbered in correspondence with the reduction in this distance; for example, point 1 has the greatest positive distance, while point 7 has the least negative distance. 
The estimates obtained enable us, in practice, to make recommendations on the arrangement of the solid-fuel packets in the braking motor. It is important that the burn-up of the fuel should lead to changes in the moments of inertia for which, first, the point of the design parameters $\left\{\Delta_{A}, \Delta_{C}\right\}$ should be situated in region $D$ and, second, should be furthest of all from the straight line (4.10).

Calculations were carried out of the nutation angle using the complete equations of motion (2.14) and (2.15), taking relation (3.8) into account for certain points $\left\{\Delta_{A}, \Delta_{C}\right\}$ from the discrete set of values (Fig. 3), which satisfy condition (4.2), for the same inertial-mass parameters and initial conditions of motion

$$
\begin{aligned}
& A_{1}=A_{2}=2.5 \mathrm{~kg} \mathrm{~m}^{2}, \quad C_{1}=0.9 \mathrm{~kg} \mathrm{~m}^{2}, \quad C_{2}=0.3 \mathrm{~kg} \mathrm{~m}^{2}, \quad \psi_{0}=\gamma_{0}=0.1 \mathrm{rad} \\
& r_{0}=0 \mathrm{rad} / \mathrm{s}, \quad \sigma_{0}=20 \mathrm{rad} / \mathrm{s}, \quad L_{0}=1.1 \mathrm{rad} / \mathrm{s}, \quad s_{0}=0 \mathrm{rad}, \quad T=25 \mathrm{~s} .
\end{aligned}
$$

The results, presented in Fig. 4, show that the least values of the nutation angle are reached for point 1 , while the greatest values are reached for point 7 from the space $\left\{\Delta_{A}, \Delta_{C}\right\}$. The calculations confirm the correctness of the main analytical conclusions, formulated in the form of conditions (4.8) and (4.9).

In conclusion, we note that the equations obtained and their approximate solutions may be useful when investigating the motion of other classes of spacecraft, representing a system of coaxial bodies of variable composition.

This research was supported by the Russian Foundation for Basic Research (03-01-00151).

\section{REFERENCES}

1. ASLANOV, V. S. and DOROSHIN, A. V., Stabilization of the descent apparatus by partial twisting when carrying out uncontrolled descent. Kosmich. Issled., 2002, 40, 2, 193-200.

2. KOSMODEM'YANSKII, A. A., Course in Theoretical Mechanics, Part 2, Prosveshcheniye, Moscow, 1966.

3. MARKEYEV, A. P., Theoretical Mechanics. Nauka, Moscow, 1990.

4. ANIKEYEV, G. I., Unsteady Almost Periodic Oscillations of Rotors. Nauka, Moscow, 1979. 